\title{
Hemoperitoneum from Ruptured Uterine Tumor Invading Ileal Mesentery and Mesosigmoid
}

\author{
Case report and literature review
}

\author{
NICOLAE BACALBASA ${ }^{1 \#}$, CASSIUS CIRLAN ${ }^{\#}$, IOAN SAS ${ }^{2}$, IRINA BALESCU ${ }^{3}$, MIHAI DIMITRIU1', MADALINA MARCU ${ }^{1}$, \\ MIHAELA MOLDOVAN ${ }^{2}$, DANIEL PIRICI ${ }^{4}$, ANDREI GHEORGHE MARIUS MOTOC ${ }^{5 *}$, DAN BOGDAN NAVOLAN ${ }^{2}$ \\ ${ }^{1}$ Carol Davila University of Medicine and Pharmacy, 8 Eroii Sanitari Blvd., 050474, Bucharest, Romania \\ ${ }^{2}$ Victor Babes University of Medicine and Pharmacy, Department of Obstetrics Gynecology, Eftimie Murgu Sq., 300041, Timisoara, \\ Romania \\ ${ }^{3}$ Ponderas Hospital, 85 A. Nicolae Caramfil, 014142, Bucharest, Romania \\ ${ }^{4}$ University of Medicine and Pharmacy, Department of Histology, 64B 1 Mai Blvd., 200638, Craiova, Romania \\ ${ }^{5}$ Victor Babes University of Medicine and Pharmacy, Department of Anatomy, Eftimie Murgu Sq., 300041, Timisoara, Romania
}

\begin{abstract}
Uterine sarcomas are rare but very aggressive gynecologic malignancies. The most frequent signs and symptoms at the moment of diagnosis include abnormal uterine bleeding or diffuse abdomino-pelvic pains while acute abdomen by uterine rupture is an uncommon situation. We present the case of a 72 year old patient who presented in emergency for diffuse abdominal pain and hypotension. An emergency laparotomy was performed and a ruptured uterine tumor with small bowel and sigmoidian serosal invasion and intraperitoneal bleeding was found. The tumor was resected en bloc with total hysterectomy, bilateral adnexectomy, segmental enterectomy and sigmoidectomy, pelvic and para-aortic lymph node dissection. The histopathological and immunohistochemistry examination revealed a poorly differentiated sarcoma. Although it is a rare condition, a uterine sarcoma can rupture and cause hemoperitoneum; emergency surgery while respecting oncologic principles of complete resection is mandatory.
\end{abstract}

Keywords: ruptured uterine tumor; uterine sarcoma; hemoperitoneum; radical surgery

Uterine sarcomas are rare tumors with poor prognosis, accounting for less than $3 \%$ of all genital tract malignancies [1]. The main histopathological types consist of pure mesenchymal tumors - leiomyosarcoma (LMS) and endometrial stromal sarcoma (ESS) and mixed epithelial and mesenchymal tumors - adenosarcoma (AS) while carcinosarcomas are considered as metaplastic forms of endometrial cancer with aggressive behavior resembling rather an uterine sarcomas [2]. The most frequently encountered symptoms are abnormal uterine bleeding $(60 \%)$, abdominal pain $(50 \%)$ while uterine rupture with hemoperitoneum at presentation is an extremely rare situation [3]. We present the case of a 72 year old patient who presented in emergency for abdominal pain in whom a ruptured uterine tumor with associated hemoperitoneum was found.

\section{Experimental part}

\section{Materials and method}

For immunohistochemistry, seriate sections were cut and collected on poly-L-lysine coated slides, deparaffinated and rehydrated in grading alcohol series. The slides were then processed for microwave antigen retrieval in citrate buffer $p \mathrm{H} 6$ for $21 \mathrm{~min}$, allowed to cool to room temperature, and then the endogenous peroxidase was blocked by incubation in 3\% water peroxide, for 30 min. Next, the unspecific antigenic binding sites were blocked in $2 \%$ skimmed milk for $30 \mathrm{~min}$, and the primary antibodies were added on the slides for an overnight incubation at 4C (table 1). The next day, the slides were washed in phosphate saline solution (PBS), the signal amplified with speciesspecific human adsorbed secondary antibodies coupled with horseradish peroxidase for 1 hour (Nikirei). The signal

\begin{tabular}{|c|c|c|c|c|c|}
\hline Name & Clonality & Dilution & Source & $\begin{array}{l}\text { Positivity of } \\
\text { the tumour }\end{array}$ & \multirow{10}{*}{$\begin{array}{c}\text { Table } 1 \\
\text { PRIMARY ANTIBODIES } \\
\text { AND REACTIVITY OF } \\
\text { TUMOR }\end{array}$} \\
\hline Carcinoembrionar antigen & Rabbit, polyclonal & $1: 200$ & $\begin{array}{l}\text { A0115, Dako, Glostrup, } \\
\text { Denmark }\end{array}$ & - & \\
\hline Citokeratin $\mathrm{AE} 1 / \mathrm{AE} 3$ & $\begin{array}{l}\text { Mouse, IgGlk, clone } \\
\text { AE1/AE3 }\end{array}$ & $1: 50$ & M3515, Dako & - & \\
\hline Desmin & Mouse, IgG1k, clone D33 & $1: 50$ & M0760, Dako & - & \\
\hline Indoleamine 2,3-dioxygenase 2 & Rabbit, polyclonal & $1: 50$ & $\begin{array}{l}\text { A.A274-303, Antibodies } \\
\text { online }\end{array}$ & ++ & \\
\hline Ki67 & Mouse, IgGlk, clone MIB-1 & $1: 100$ & M7240, Dako & ++ & \\
\hline p53 & Mouse, IgG2b, clone DO-7 & $1: 50$ & M7001, Dako & ++ & \\
\hline Smooth muscle actin & Mouse, IgG2a, clone 1A4 & $1: 100$ & M0851, Dako & - & \\
\hline Tryptophan 2,3-dioxygenase 2 & Rabbit, polyclonal & $1: 100$ & $\begin{array}{l}\text { NBP2-02002, Novus } \\
\text { Biological }\end{array}$ & + & \\
\hline Vimentin & Mouse, IgG1k, clone V9 & $1: 100$ & M0725, Dako & ++ & \\
\hline
\end{tabular}

*email: amotoc@umft.ro; Phoneno: +40722277806; 
was lastly detected using 3.3'-Diaminobenzidine (DAB) (Nikirei) and the reaction was stopped in PBS. Then the sections were counterstained in Mayer's Hematoxylin, dehydrated in increasing alcohols, cleared in xylene and mounted with permanent mounting medium (Merck). Slides were viewed and images captured on a Nikon $55 i$ microscope (Elta 90, Bucharest, Romania) equipped with a 5 megapixel color cooled CCD camera and the Image ProPlus AMS 7 software (Media Cybernetics, Rockville, MD, USA).

\section{Case report}

A 72 year old patient presented in emergency unit for abdominal pain with sudden on-set $12 \mathrm{~h}$ before; the clinical examination revealed signs of acute abdomen associated with the presence of a large pelvi-abdominal tumor; general signs of shock including tachycardia and hypotension were also encountered.

\section{Description of surgery and macroscopic findings}

Intraoperatively a large ruptured uterine tumor was found with direct invasion of the small bowel mesentery and the mesosigmoid associated with hemoperitoneum originating from the uterine tumor (fig. $1 \mathrm{~A}-\mathrm{F}$ ). The tumor was resected en bloc with total radical hysterectomy and bilateral adnexectomy, segmentary distal enterectomy and sigmoidectomy; the continuity of the digestive tract was established by an end-to end entero-enterostomy and by a end-to-end colo-rectal anastomosis; enlarged ganglia were identified in the aorto-caval territory and bilaterally along the common iliac vessels, thus inter-aortico-caval and pelvic lymph node dissection was also performed (fig. 2A$\mathrm{E})$. The patient was subsequently referred to the oncology department for adjuvant chemotherapy.
A)

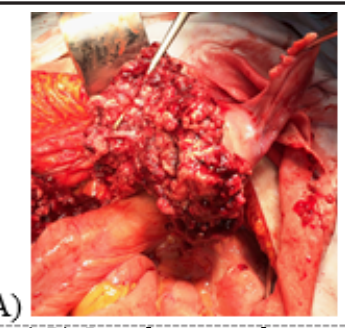

A,B) Initial aspect: Targe ruptured

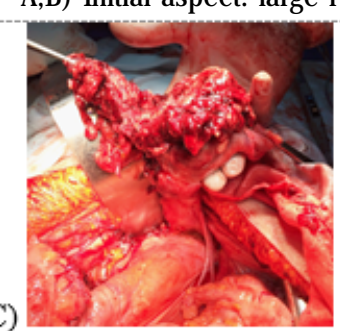

C) Aspect after complete mobilization of the tumor en bloc with total

hysterectomy and bilateral adnexectomy.

E)

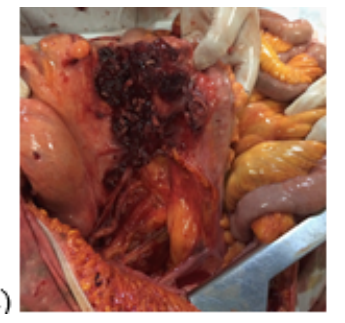

B)

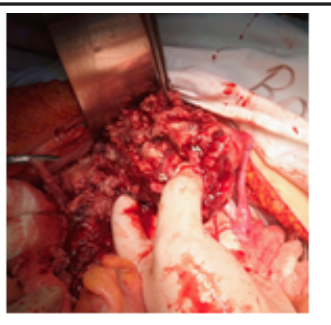

D)

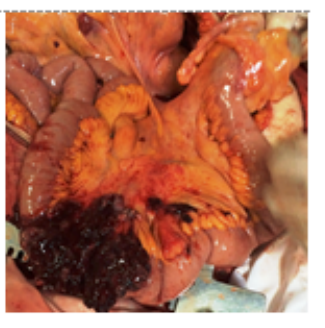

D) Tumoral invasion of the mesentery of an ileal loop

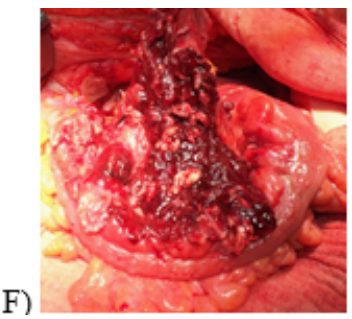

$E, F)$ tumoral involvement of the sigmoid

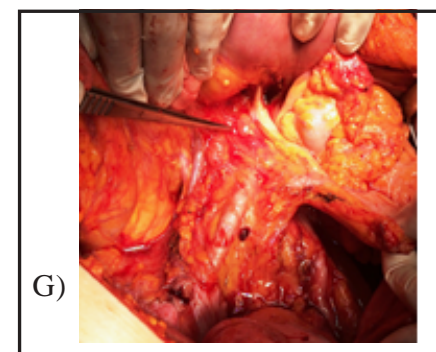

G) Enlarged lymph nodes on the anterior surface of the abdominal aorta

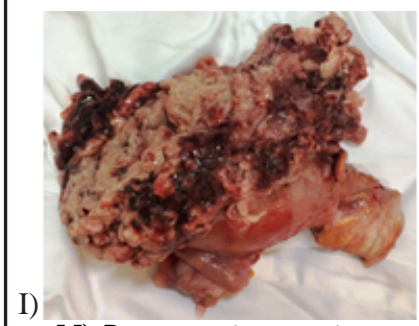

I,J) Postoperative specimen: total hysterectomy with bilateral adnexectomy presenting a large ruptured uterine tumor

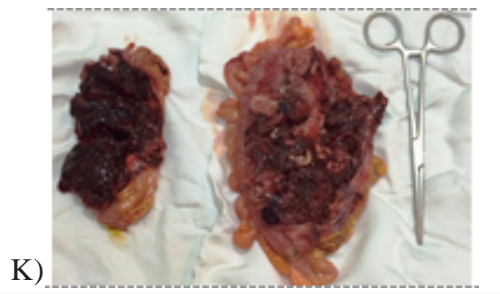

K) Postoperative other two specimens: segmental enterectomy and sigmoidectomy presenting tumoral invasion.
Fig. 1. Intraabdominal view of uterine tumor $(A-C)$ with imvasion of mesenter and sigmoid (D-F), lymphnode involvment (G-H) and postoperative specimens (I-K)

\section{Description of histopathological results}

The histopathological examination confirmed the presence of a poorly differentiated sarcoma (fig. 2 A-D).
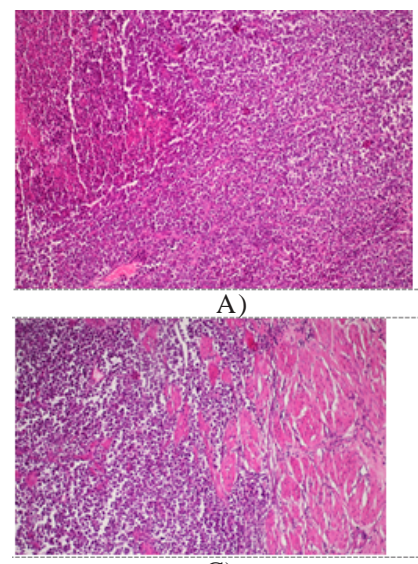

C)
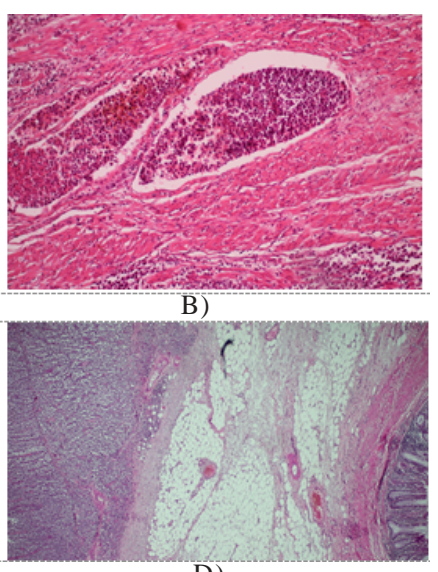

D)
Fig. 2 (a-d). Histopathological evaluation (40x) of hematoxilin eosin stained tissue showed a poorly differentiated sarcoma (a) with tumoral emboli (b), invasion of miometrum (c) and of the intestinal wall $(d)$

Description of immunohitoshemistry findings

Tumor cells were intensely positive for vimentin (fig 3 A, B) in their cytoplasm, with the entire population expressing it to different extents. SMA was completely negative in the tumor cells' mass, with only blood vessels walls being immunopositive in the tumor stroma (fig $3 \mathrm{C}$, D). Tumor stroma was infiltrated by a moderate mass of lymph cells and macrophages, as illustrated by immunohistochemistry for CD68 (fig $3 \mathrm{E}, \mathrm{F}$ ). Around 20\% 

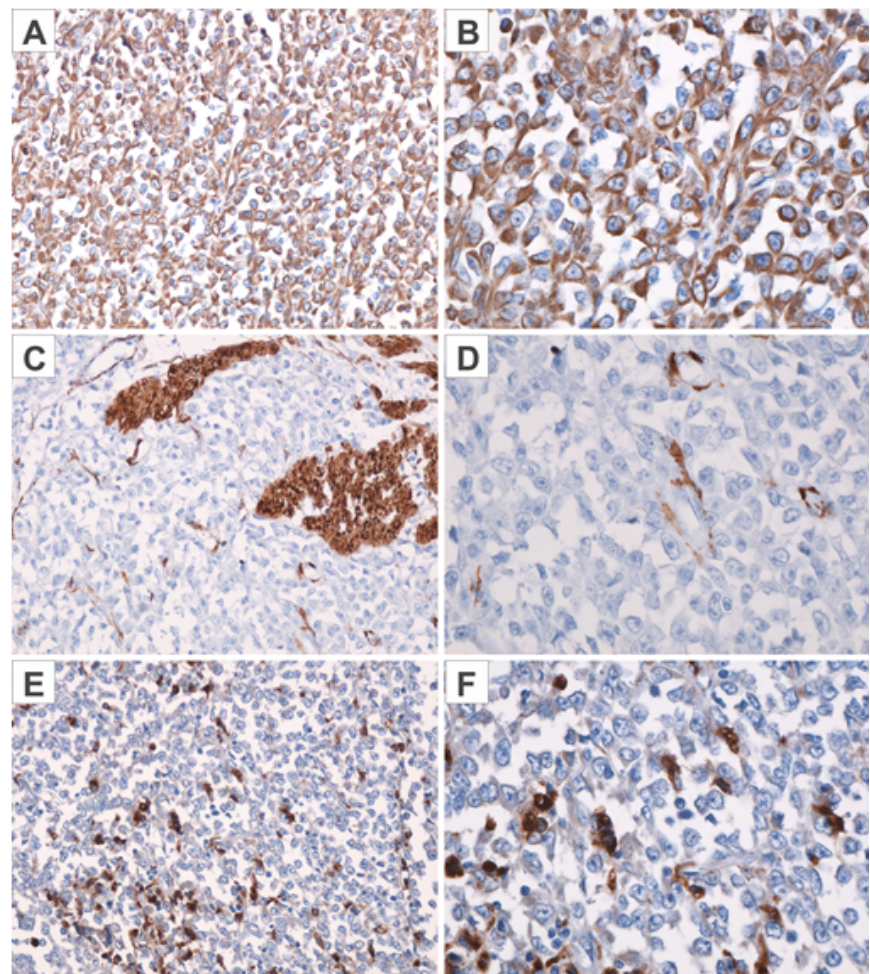

Fig. 3. Immunohistochemistry stainging of the tumor with IDO 2vimentin $(A, 20 x)(B, 40 x)$, SMA $(C, 20 x)(D, 40 x)$ and $C D 68(E, 20 x)$ $(\mathrm{F}, 40 \mathrm{x})$

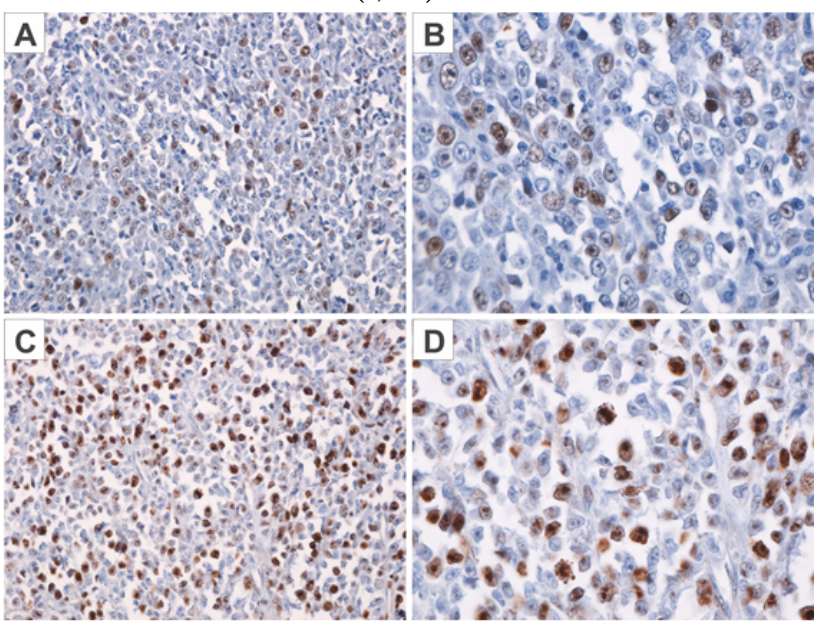

Fig. 4. Immunohistochemistry stainging of the tumor with p53

$(A, 20 X)(B, 40 X)$ and $K I 67(C, 20 X)(D, 40 X)$

of the tumor cells were moderately immunopositive for p53 (fig 4A, B), the aggressive pattern being also underlined by a much higher positivity percentage for ki67 ( 50\% of the tumor cells) (fig 4 C, D).

IDO2 was moderately positive in the cytoplasm of the tumor cells, in almost all of the non-stromal cells, the immunostaining pattern being vesicular-like in its appearance (fig $5 \mathrm{~A}, \mathrm{~B}$ ). The adjacentnon-invaded intestinal mucosae exhibited this marker however with a much higher intensity in the cytoplasm of the enterocytes, but not in the microvilli (fig 5 C, D).

TDO2 had a different expression pattern in the tumor mass, being expressed in less than $10 \%$ of the tumor cells, as small granule and dots in the cytoplasm. (fig 6 A, B). The adjacent non-invaded intestinal mucosae exhibited this marker like dense-diffuse dots in the cytoplasm of the enterocytes and in the upper portion of the intestinal glands (fig 6C, D).
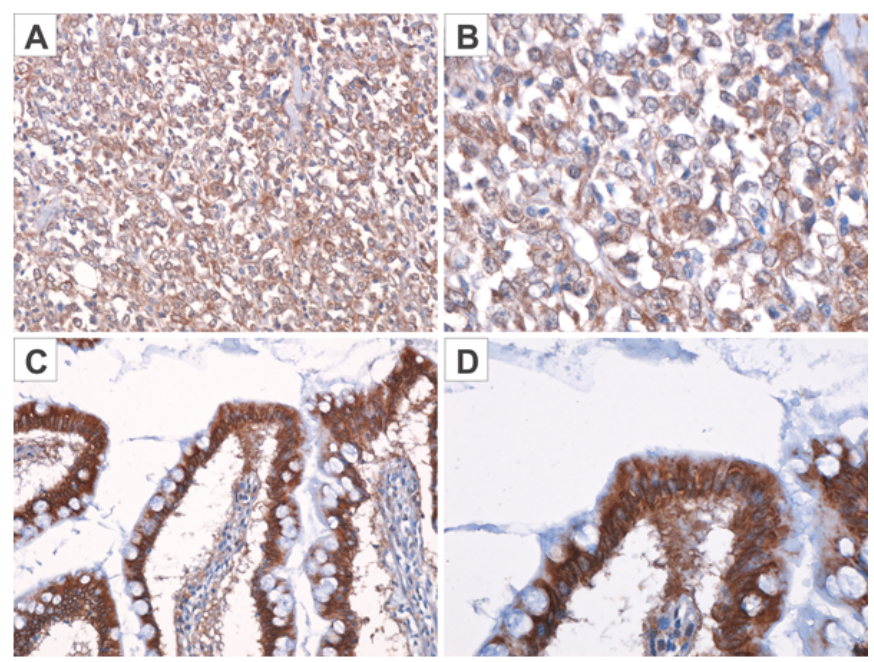

Fig. 5. Immunohistochemistry stainging with IDO2 of tumor cells $(A, 20 X), B(40 X)$ and intestinal wall cells $(C, 20 X),(D, 40 X)$

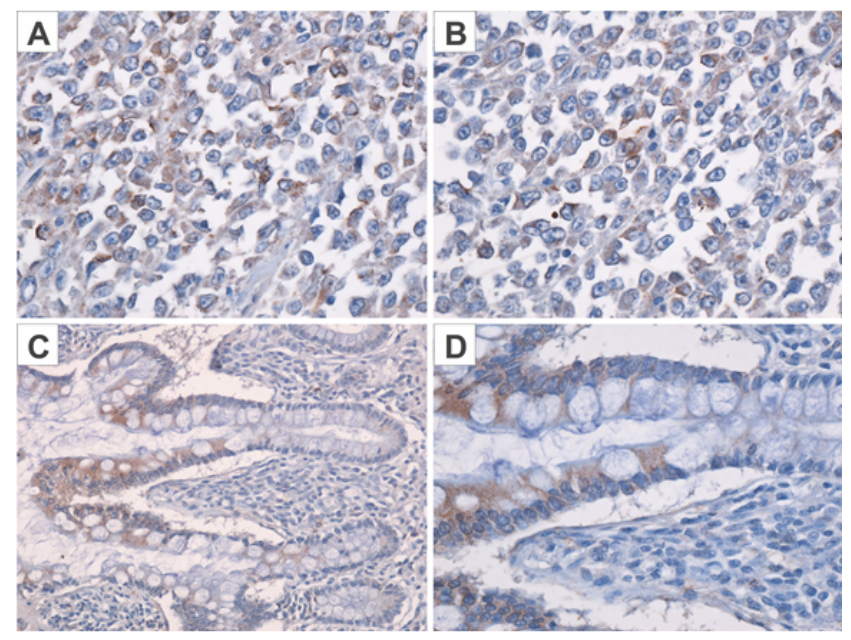

Fig. 6. Immunohistochemistry stainging with TDO2 of tumor cells $(A, 20 X), B(40 X)$ and intestinal wall cells $(C, 20 X),(D, 40 X)$

\section{Results and iscussions}

Uterine sarcomas usually appear in patients submitted to an importanthormonal stimulation due to the existence of various ovarian tumors or due to the prolonged administration of oral contraceptives which seem to increase the rate of sarcomatous degeneration of the mesothelial and submesothelial cells $[2,4]$. We have identified only few reports describing cases of hemoperitoneum caused by ruptured uterine tumors, and most of them appeared in benign conditions [4-6]. Ozcan et al. [7] presented the case of a 14 year old patient diagnosed initially with a uterine tumor which was resected, the histopathological findings reporting a benign spindle cell leiomyomata uteri; eight months later she returned for abdominal and pelvic pain associated with vomiting and dyspnea. Re-laparotomy was performed and disseminated pelvic and abdominal tumors were seen associated with a large uterine ruptured tumor. They were all resected and the histopathological findings revealed adenosarcoma with stromal overgrowth. Arneja et al. [8] reported a similar case in a 18 year old female who had undergone several laparoscopic surgical resections of uterine leiyomyomas. In this case the histopatological reportshowed a peritonealy disseminated leiomyomatosis; peritoneal dissemination in a benign tumor was explained by the presence of smooth muscle metaplasia of subperitoneal mesenchymal stem cells of the secondary müllerian system. 
In our case the histopathological studies revealed the presence of a poorly differentiated uterine sarcoma. It is well known that this histopathological sub-type is the most encountered sarcoma of the uterus, accounting for almost $40 \%$ of all uterine sarcomas [2]. Similarly to other highgrade sarcomas, it has a high capacity to early metastasize via hematogenous route, primarly to the lungs or liver; the lymphatic route of dissemination is uncommon, positive lymph nodes being reported in less than $3 \%$ of cases [9]. While cases presenting with low-grade stromal sarcomas usually report a slow rate of growth and a tendency of uterus confined disease in more than $70 \%$ of cases, high grade stromal sarcomas behave much more aggressively, spreading by local extension or abdominal implantation $[2,9]$. When it comes to the most appropriate treatment option, cases presenting advanced disease are rather candidates for systemic chemotherapy or targeted agents, usually with palliative intent $[2,10]$. Hemoperitoneum as the first sign at presentation of an uterine sarcoma is a very rare and life-threatening condition; emergency surgery in order to perform hemostasis is mandatory; however once the surgical emergency is resolved the lesion must be approached according to oncological principles of complete resection as it brings the only chance of best long-term survival benefit. Even during an emergency laparotomy in an elderly patient we performed a multivisceral resection with associated extended lymph node dissection as the biological status of the patient seemed to allow it. Although rare, the possibility of hemoperitoneum expressing an underlying gynecologic malignancy is possible and emergency laparotomy of hemostasis is mandatory while respecting surgical oncology principles in relation to the severity of the hemoperitoneum, extent of lesions and biological condition of the patient.

Acknowledgments:This work was supported by a grant of the Victor BAbes UNiversity of Medicine and Pharmacy Timisoara, project number ONCOPREDICT - nr. 7198 P-IV-CI-PDCC 2015/2016/01.07.2015

\section{References}

1.SEDDON, B.M., DAVDA, R. Eur J Radiol. 2011;78:30-40.

2.D'ANGELO, E., PRAT, J. Gynecol Oncol. 2010;116:131-139.

3.IP, P.P., CHEUNG, A.N. Best Pract Res Clin Obstet Gynaecol. 2011;25:691-704.

4.TAKAI, H., TANI, H., MATSUSHITA, H. J Reprod Med. 2013;58:72-74. 5.EKANE, G.H., TEBEU, P.M., OBINCHEMTI, T.E., NJAMEN, T.N., NGUEFACK, C.T., KAMGAING, J.T. et al. The Pan African Medical J ournal. 2013;16:57

6.CLAEYS, J., HELLENDOOM, I., HAMERLYNCK, T., BOSTEELS, J., WEYERS, $S$. The risk of uterine rupture after myomectomy: a systematic review of the literature and meta-analysis, Gynecological Surgery. 2014;11:197-206

7.0ZCAN, J. et al. Oncol. Rep. (2014), http:// dx.doi.org/10.1016/ j.gynor.2014.05.004

8. ARNEJA, S.K. J Pediatr Adolesc Gynecol. 2011;24:e73-e76.

9.GIUNTOLI, R.L., METZINGER, D.S., DIMARCO, C.S. et al. Gynecol Oncol. 2003;89:460-469.

10.HENSLEY, M.L., ISHILL, N., SOSLOW, R. et al. Gynecol Oncol. 2009;112:563-567.

Manuscript received: 16.05 .2018 